\title{
El análisis de errores como herramienta para el proceso de enseñanza-aprendizaje
}

\author{
Lilian Paola Umbarila V. \\ Recibido el 03 de julio de 2009. Aprobado el 22 de octubre de 2009
}

\begin{abstract}
Resumen
En el presente articulo, se realiza un análisis de los diferentes errores en que los estudiantes incurren en el proceso de aprendizaje del Algebra, y pretende contribuir al proceso de enseñanza-aprendizaje, tanto para profesores como para alumnos, buscando convertirlos en una herramienta que cuestione los conocimientos adquiridos, con el fin de lograr una mayor comprensión y familiaridad con el razonamiento algebraico.
\end{abstract}

\section{Palabras clave:}

Aprendizaje, álgebra, errores en el aprendizaje, estrategias metodológicas, deserción estudiantil, dificultad, estudiante, docente.

\begin{abstract}
In this paper, be performed an analysis of different errors that students engage in the learning process algebra, and seeks contributing to the teaching-learning process for both teachers and learners, seeks to become them a tool to question knowledge gained, with the aim of achieving greater understanding and familiarity with algebraic reasoning.
\end{abstract}

\section{Keywords}

Learning, algebra, learning mistakes, methodological strategies, student desertion, difficulty, student, teacher.

\section{Introducción}

Uno de los grandes retos de la actualidad es mejorar la calidad de la educación. Procurar que los estudiantes aprendan más, usando los medios adecuados es la preocupación de docentes e instituciones educativas. Para ello, es necesario que los alumnos cuenten con ambientes de aprendizaje efectivos y didácticos; entornos educativos que les permitan desarrollar sus habilidades para pensar y su capacidad para aprender.

En la transición de la educación media a la educación superior, la formación matemática es un factor decisivo entre los estudiantes que aprueban o reprueban, convirtiendo a ésta materia en primer semestre como factor de alta incidencia en la deserción estudiantil. 
El proceso de aprendizaje del álgebra genera un sinnúmero de dificultades a los estudiantes; éstas, tienen que ver con la complejidad en la resolución de problemas haciendo uso de los objetos del álgebra, el pensamiento abstracto y las actitudes afectivas y emocionales. Como consecuencia, se manifiestan a través de sentimientos de tensión y miedo hacia la materia. Los modos de proceder y actuar en el pensamiento algebraico provocan rupturas que se convierten en dificultades en el desarrollo normal de construcción del conocimiento matemático. Como consecuencia, estas se concretan en la práctica en forma de obstáculos y se manifiestan mediante errores. En algunos casos los estudiantes crean nuevos procesos omitiendo la rigidez matemática de los mismos, llevando estos, a respuestas incorrectas en las que el estudiante es incapaz de reconocer donde se encuentra el error. La respuesta del docente en la gran mayoría de los casos se simplifica a calificar como deficiente la capacidad del estudiante para realizar procedimientos sin tener en cuenta la corrección oportuna de los mismos, lo cual, agudiza en la estructura cognitiva del estudiante retrasos fortaleciendo algoritmos mal aprendidos los que a su vez, se reflejan en su rendimiento académico y posteriores aprendizajes. Lo anterior, genera desconcierto en el estudiante, quien pierde confianza y ánimo ante la recurrencia de estos episodios.

En este sentido, la gran mayoría de los estudiantes cuando acceden a la educación superior, específicamente, al iniciar carreras de ingeniería, generalmente desertan en los primeros semestres debido al bajo rendimiento en las materias referidas a las ciencias exactas y/o cambian su inclinación hacia carreras donde la existencia de matemáticas en su currículo no resulte tan determinante.

Las dificultades que se generan entorno al aprendizaje del álgebra, no se pueden evitar ya que forman parte del proceso normal de construcción del conocimiento matemático, y es allí, donde los profesores deben aprender a reconocerlas y reflexionar acerca de ellas para incidir positivamente en el proceso de enseñanza-aprendizaje.

La interpretación y análisis de los errores cometidos por los estudiantes permite enriquecer los procesos de la educación matemática proveyéndola de información acerca de la forma en que los estudiantes interpretan los problemas y resuelven los diferentes procedimientos algebraicos. Esta información es una forma de ayudar a los alumnos a corregir errores y señala las posibles causas de las dificultades para que aprendan álgebra.
Determinar los errores más comunes que cometen los estudiantes, facilita a los docentes el desarrollo de estrategias académicas, con el fin de garantizar la efectividad del proceso de enseñanza aprendizaje en el aula.

\section{Desarrollo de la temática}

Desde éste punto de vista, es importante analizar el concepto de error en la apropiación del conocimiento y las construcciones teóricas que algunos autores han generado alrededor de éste tema, con la intención de afianzar la solidez en el descubrimiento temprano de los mismos.

Así, Moliner (citado en Cañadas \& Castro (2001) define el error como "el desacierto o equivocación en cierta cosa". Lo anterior sugiere que los errores son posibilidades factibles dentro del aprendizaje, de modo que éstos representan en si mismos, la garantía del proceso intangible al interior del sujeto, es decir, la relación entre los conceptos y los resultados.

En esta misma línea, Borasi (citado por Cañadas \& Castro (2001) refiere que: "los errores en el proceso de enseñanza aprendizaje son importantes por que permiten conocer la naturaleza de las nociones matemáticas fundamentales", es decir, el error es la posibilidad objetiva de identificar y valorar los conceptos adquiridos durante la interacción con el conocimiento, de modo que, pueda resaltarse el nivel de ejecución realizado por la persona; por ejemplo: el estudiante puede realizar una operación matemática elevando $2^{3}$ ante lo cual una respuesta equivocada sería "igual a 6", esto podría sugerir que no tiene claro el concepto de potenciación, si se centra la atención en el resultado indicado por el alumno.

Por otro lado, si se analiza el progreso de las ciencias puras durante la evolución del hombre, se nota que en éste, ha sido determinante el concepto de error. Cometer errores ha permitido establecer la aparición de leyes, teoremas e hipótesis, que después de ser probados una y otra vez, ha conllevando a la aparición de procedimientos objetivos que validen supuestos o acciones.

Bien lo ilustra Rico (1995) al referirse a lo postulado por Popper, en el que éste reconoce la importancia de explicar la falsedad advirtiendo que: "la verdad es siempre reconocible como verdad, sino es así, solo es necesario desvelar esa verdad o descubrirla". Entonces, Popper defiende que no es posible establecer la verdad manifiesta desde una sola perspectiva, ya que ésta es adyacente tanto a las experiencias propias del individuo en relación con su forma de 
conocimiento, como a las diversas formas de aprendizaje de otros colectivos.

Las reflexiones de Rico con respecto a Popper, coinciden en el ámbito matemático, en relación a la explicación del error. Es necesario partir del hecho que el conocimiento en sí, depende de las diferentes formas en las que el individuo aprende, y por ende, la alteración (entendida como error) de dicha comprensión es el resultado de la condición humana. De igual forma, es pertinente reafirmar que el error es condición necesaria para acceder al conocimiento, como ya se había mencionado, de forma tal, que permite la consecución de una verdad objetiva, a través de la retroalimentación permanente.

Por su parte, la teoría constructivista ratifica que la noción de error es parte del conocimiento, estableciendo que deben desarrollarse paralelamente procesos de construcción, que permitan el establecimiento de causalidades y posibles alternativas de corrección, para lo cual pueden utilizarse acciones formativas de carácter objetivo sobre los resultados obtenidos.

La corriente teórica cognitivista, sostiene que el estudiante posee conocimientos previos, procedentes de anteriores saberes que, al conjugarse con los nuevos que se pretenden adquirir, pueden generar bloqueos cognitivos manifestados a través de errores que impiden o dificultan su proceso de aprendizaje. Es por esto, que se plantea la necesidad de detectar, analizar e intervenir sobre los mimos, con el fin de garantizar el aprendizaje significativo de las matemáticas.

Al respecto, autores como Brausseau, Davis y Werner citados por Engler (2004) sugieren que los estudiantes construyen su conocimiento, en algunas circunstancias, de forma independiente a las explicaciones del profesor, razón por la cual dichas construcciones pueden ser equivocadas.

Con el fin de identificar los errores cometidos por algunos estudiantes, estos tres autores realizaron un registro de información a partir de la observación del trabajo con alumnos en el aula, concluyendo que:

a. El error termina siendo la repetición sistémica de cualquier proceso con la plena creencia de ser correcto.

b. Los errores suelen mantenerse debido a que, de forma circunstancial, los profesores no se percatan de la comisión de los mismos por parte de los estudiantes.

c.La destreza de los estudiantes permite en ocasiones, desarrollar metodologías que facilitan el establecimiento y mantenimiento de dichos errores.
Algunos autores como Davis, citado por Rico (1995) se han propuesto elaborar taxonomías de los errores que se cometen en el aprendizaje del álgebra de acuerdo a investigaciones hechas por ellos mismos.

La propuesta de Davis, postula:

1. Revisiones binarias. Ejemplo: $-22=4$

2. Errores por el lenguaje o la notación: $3 X+2 X^{2}=5 X^{3}$

3. Errores por recuperación de un esquema.

4. Errores producidos por una representación inadecuada. Estos se presentan cuando se asume a una letra como incógnita, cuando su significado real es una variable.

5. Reglas que producen reglas. Así de las implicadas que sí: $2^{2}=4$ y $3^{2}=9$ entonces $(2+3)^{2}=4+9$

Lo anterior refiere que, los errores en álgebra no están expuestos únicamente por el aprendizaje inmediato, sino por los procesos mal aprendidos en aritmética. Con el fin de puntualizar sobre las características de los errores más recurrentes, Rico cita a Muherg (1989), quien refiere que:

- Los errores aparecen de forma inesperada o "sorprendente", si se tiene en cuenta que el docente ha omitido su manifestación de forma involuntaria o por situaciones coyunturales.

- De igual forma son persistente, debido probablemente, a que el estudiante los ha interiorizado haciéndolos parte de sus propias construcciones, para lo cual, es necesario desarrollar técnicas que favorezcan la reestructuración cognitiva de dichos conceptos.

-Pueden jerarquizarse en sistemáticos o por azar. Los primeros refieren que la comprensión que ha hecho el estudiante dentro de su proceso de pensamiento es equivocada, convirtiéndose en un acción mecánica. Por su parte, los segundos son resultado de deficiencias atencionales o coyunturales que no tiene mayor relevancia.

-Finalmente, los errores pueden producirse por la omisión del significado de símbolos y conceptos necesarios en las operaciones a realizar.

Dentro de esta dinámica taxonómica, Pochulu (2005) identifica las siguientes características del error en el aprendizaje de la matemática:

-El uso reiterativo de procedimientos operacionales que carecen de justificación teórica.

- Manejo de reglas sin trascendencia que pueden dilatar el proceso de aprendizaje de la matemática.

-Poca recursividad para llevar lo real a lo abstracto dándole aplicabilidad en la resolución de problemas. 
-No se enfatiza sobre las competencias interpretativa y argumentativa, a lo que Pochulu (2005) añade: "lectura critica de datos y análisis de gráficos".

-Saturación de imágenes y diseños gráficos para la conceptualización.

- La solución de los problemas se centra en competencias numéricas.

De lo anterior, se puede concluir que la responsabilidad en la aparición de los errores no recae solamente sobre los estudiantes, sino que también factores exógenos como docentes, ambientes de aprendizaje, textos y el entorno familiar entre otros, son relevantes en la comisión de los mismos.

Otro autor muy reconocido en el análisis de errores en el aprendizaje del álgebra es Radatz (1980), quien propone que los errores se pueden clasificar como sigue:

1.Errores debidos a dificultades en el lenguaje.

2.Errores debidos a dificultades para obtener información espacial.

3.Errores debidos a aun aprendizaje deficiente de destrezas, hechos y conceptos previos.

4.Errores debidos a asociaciones incorrectas o a rigidez del pensamiento.

5.Errores debidos a la aplicación de reglas o estrategias irrelevantes.

De ésta manera, se ratifica la importancia de estudiar, analizar e investigar los errores que se presentan en el aprendizaje, con el fin de reconocer por qué, cómo y cuándo se manifiestan, así como la necesidad de desarrollar herramientas pedagógicas preventivas que faciliten la detección de errores, además de proponer acciones de intervención matemática que minimicen la aparición de los mismos.

\section{Conclusiones}

El error hace parte de todo conocimiento. Es inherente al mismo, y se encuentra presente en todos los procesos de aprendizaje a los cuales es expuesto el ser humano.

- De acuerdo a lo expresado por los autores referidos en el articulo, es posible evidenciar que los errores son producto también de acciones circunstancia- les, en las cuales juegan variables culturales, sociales y pedagógicas adyacentes tanto al proceso educativo como a los involucrados en el mismo (estudiante - docentes). De ésta manera, se sugiere contemplar estrategias de prevención de éstos errores en el diseño y construcción de currículos académicos de Educación Media, con el fin de potenciar los procesos de aprendizaje en los estudiantes de Educación Superior.

\section{Sugerencias:}

Para fortalecer los procesos de enseñanza y aprendizaje del algebra y la inclusión de la comisión de los errores de los estudiantes en el mismo, se pueden tener en cuenta las siguientes sugerencias adicionales:

Permitir desde la educación superior mayores espacios académicos fuera de clase, para preparar a los estudiantes que inician, en manejo de conceptos básicos de la educación media, que fueron olvidados tempranamente.

Enseñar los procedimientos y operaciones conceptualizándolos y contextualizándolos, con el fin de que el estudiante encuentre sentido al aprendizaje del álgebra.

\section{Referencias}

[1] Cañadas, M. \& Castro, E. (2002), Errores en la resolución de problemas matemáticos de carácter inductivo. disponible en: http://ddm.ugr.es/gpnumerico/ numerico es.html, recuperado: 1 de julio de 2008.

[2] Engler, A. E† al. (2004). Los errores en el aprendizaje de matemática, Revista Premisa, año 6, núm 23, (pp.3-16), recuperado el 8 julio de 2008, de http:/l www.soarem.org.ar/revistapremisa.htm

[3] Pochulu, M. (2008). Análisis y categorización de errores en el aprendizaje de la matemática en alumnos que ingresan a la universidad. Revista lberoamericana de Educación, recuperado el 4 de julio de 2008, de http://www.rieoei.org/deloslectores, de.

[4] Radatz, H. (1980), Students' errors in the mathematical learning process: a survey. For the Learning of Mathematics, V. 1, No 1, pp. 16-20.

[5] Rico, L. (1995). Errores en el aprendizaje de las Matemáticas. En Kilpatrick, J.; [6] Rico, L. \& Gómez, P. Junio de 2008 . Educación Matemática. G.E.I. Bogotá. 69-108.

Lilian Paola Umbarila Valencia. Ingeniera Química, candidata al título de "Magister en Investigación y Docencia Universitaria", Universidad Sergio Arboleda 2009, "Especialista en Investigación y Docencia Universitaria", Universidad Sergio Arboleda 2008. En la actualidad, es docente del Departamento de Ciencias Básicas de la Corporación Universitaria Minuto de Dios (UNIMINUTO). lumbarila.umbarila@gmail.com 